\title{
Erratum: Ultimate Precision of Adaptive Noise Estimation [Phys. Rev. Lett. 118, 100502 (2017)]
}

\author{
Stefano Pirandola and Cosmo Lupo \\ (Received 24 August 2017; published 20 September 2017)
}

DOI: 10.1103/PhysRevLett.119.129901

In this Erratum we do not point out any error but we make some clarifications about results presented in our Letter in order to avoid possible misunderstandings by the reader. In fact, while our results were and remain correct, we have also noticed that we have left some (natural) assumptions implicit in parts of our Letter and these might be missed by the reader.

In our Letter we have considered the adaptive estimation of a noise parameter $\theta$. This is meant to be an environmental parameter, i.e., a parameter labeling the environmental state which intervenes in a dilation or simulation of the quantum channel. Such an assumption is also implicit in the simulation of a $\theta$-dependent programmable channel $\mathcal{E}_{\theta}$. For this reason, when we specify Eq. (4) of the Letter for a $\theta$-dependent programmable channel $\mathcal{E}_{\theta}$, this equation is meant to become $\mathcal{E}_{\theta}(\rho)=\mathcal{U}\left(\rho \otimes \sigma_{\mathcal{E}_{\theta}}\right)$, where $\theta$ is encoded in the program state $\sigma_{\mathcal{E}_{\theta}}$ but not in the general trace-preserving quantum operation $\mathcal{U}$.

Similarly, the same assumption is implicit in the teleportation simulation. This means that, when we specify Eq. (3) of the Letter for a $\theta$-dependent teleportation-covariant channel $\mathcal{E}_{\theta}$, this equation explicitly becomes

$$
\mathcal{E}_{\theta}(\rho)=\mathcal{T}\left(\rho \otimes \rho_{\mathcal{E}_{\theta}}\right),
$$

where $\theta$ is encoded in the channel's Choi matrix $\rho_{\mathcal{E}_{\theta}}$ but not in the teleportation LOCC $\mathcal{T}$ (with an asymptotic formulation in the bosonic case). This is indeed the type of simulation that we have used in our computations and led us to derive the "stretching" in Eq. (6) of our Letter where $\bar{\Lambda}$ is a $\theta$-independent CPTP map.

To ensure the validity of the teleportation simulation in Eq. (1) above, it is sufficient that the unknown channel $\mathcal{E}_{\theta}$ satisfies the condition of teleportation covariance [1-6] as in Eq. (2) of our Letter, where the output unitaries $V$ do not depend on the noise parameter $\theta$, i.e.,

$$
\mathcal{E}_{\theta}\left(U \rho U^{\dagger}\right)=V \mathcal{E}_{\theta}(\rho) V^{\dagger} .
$$

In other words, it is sufficient that the ensemble of channels $\left\{\mathcal{E}_{\theta}\right\}$ is jointly teleportation covariant, i.e., teleportation covariant with the same set of output correction unitaries. This is the correct way in which the reader should read Eq. (2) of our Letter and the consequent results in theorems 2 and 3.

[1] S. Pirandola, R. Laurenza, C. Ottaviani, and L. Banchi, Fundamental limits of repeaterless quantum communications, Nat. Commun. 8, 15043 (2017). See also S. Pirandola, R. Laurenza, C. Ottaviani, and L. Banchi, Fundamental limits of repeaterless quantum communications, arXiv:1510.08863.

[2] S. Pirandola and R. Laurenza, General benchmarks for quantum repeaters, arXiv:1512.04945.

[3] S. Pirandola, Capacities of repeater-assisted quantum communications, arXiv:1601.00966.

[4] R. Laurenza and S. Pirandola, General bounds for sender-receiver capacities in multipoint quantum communications, arXiv:1603.07262 [Phys. Rev. A (to be published)].

[5] R. Laurenza, S. L. Braunstein, and S. Pirandola, Finite-resource teleportation stretching for continuous-variable systems, arXiv:1706.06065.

[6] T. P. W. Cope, L. Hetzel, L. Banchi, and S. Pirandola, Simulation of non-Pauli channels, Phys. Rev. A 96, 022323 (2017). 